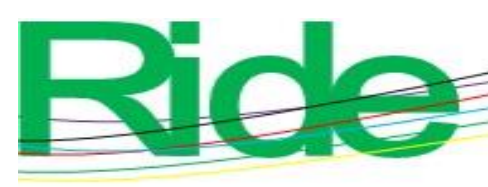

Revista Iberoamericana para la

Investigación y el Desarrollo Educativo ISSN $2007-7467$

\title{
Conceptos de éxito y fracaso desde la perspectiva de estudiantes para profesor en formación Concepts of success and failure from the perspective of students for teacher in training Conceitos de sucesso e fracasso na perspectiva dos alunos para o professor em formação
}

\author{
Galo Emanuel López Gamboa \\ Universidad Autónoma de Yucatán. Yucatán, México \\ galo.lopez@correo.uady.mx \\ https://orcid.org/0000-0001-5581-7489 \\ Edith J. Cisneros-Cohernour \\ Universidad Autónoma de Yucatán. Yucatán, México \\ ecohernour@gmail.com \\ http://orcid.org/0000-0003-2319-1519 \\ Ángel Martín Aguilar Riveroll \\ Universidad Autónoma de Yucatán. Yucatán, México \\ aguilarr@correo.uady.mx \\ https://orcid.org/0000-0001-7247-2224
}

\section{Resumen}

En esta investigación se analiza cómo se construyen las representaciones sociales que el colectivo docente en formación tiene en relación con los conceptos de éxito o fracaso escolar. Se parte desde el momento de la formación del profesorado, pues es en esta etapa donde la conformación de habitus se consolida a partir de las primeras experiencias prácticas, de la convivencia con docentes experimentados y del intercambio con el grupo de pares en formación. Para recabar los datos, se diseñó un instrumento para el análisis de representaciones sociales de profesores en formación, el cual estuvo constituido por una 
escala de diferencial semántico que se les presentó a los participantes con el fin de determinar los constructos alumno exitoso y alumno fracasado. Los resultados hallados permiten concluir que existen consensos en la representación social del éxito y del fracaso escolar orientada al esfuerzo continuo y al trabajo perseverante. Estos resultados ofrecen nuevas posibilidades para seguir indagando en este fenómeno, el cual se puede comparar con otras poblaciones y a partir de otras maneras de entender dichos conceptos.

Palabras clave: éxito escolar, formación de profesores, fracaso escolar, representaciones sociales.

\section{Abstract}

This research analyzes how the social representations that the teaching group in formation are constructed, have regarding the concepts of school success or failure. It starts from the moment of teacher training, because it is there where the conformation of habitus is consolidated due the first practical experiences, the coexistence with experienced teachers and the exchange with the peer group in formation as well.

To collect the data, an instrument was designed for the analysis of social representations of teachers in training, which was constituted by a scale of semantic differential that was presented to the participants in order to determine the social representation of successful and unsuccessful student. The results found allow us to conclude that there are consensus in the social representation of success and school failure oriented to continuous effort and persistent work. These results offer new possibilities to continue investigating this phenomenon, which can be compared with other populations and from other ways of understanding these concepts.

Keywords: school success, teacher training, school failure, social representations. 


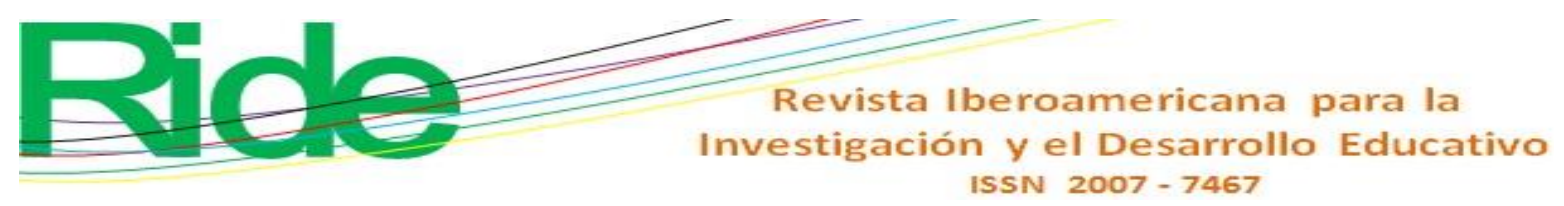

\section{Resumo}

Nesta pesquisa analisamos como são construídas as representações sociais que o grupo de ensino em formação tem em relação aos conceitos de sucesso ou fracasso escolar. Começa a partir do momento da formação de professores, pois é nesse estágio que o habitus se consolida a partir das primeiras experiências práticas, convivendo com professores experientes e trocando com o grupo de pares em formação. Para coletar os dados, foi elaborado um instrumento para a análise das representações sociais dos professores em formação, constituído por uma escala de diferencial semântico que foi apresentada aos participantes para determinar os construtos aluno de sucesso e aluno malsucedido. Os resultados encontrados nos permitem concluir que há consenso na representação social do sucesso escolar e do fracasso escolar orientado ao esforço contínuo e ao trabalho persistente. Esses resultados oferecem novas possibilidades para continuar investigando esse fenômeno, que pode ser comparado com outras populações e com outras formas de entender esses conceitos.

Palavras-chave: sucesso escolar, formação de professores, fracasso escolar, representações sociais.

Fecha Recepción: Mayo 2018

Fecha Aceptación: Noviembre 2018

\section{Introducción}

La teoría de las representaciones sociales (RS) ha tenido diferentes aplicaciones y se ha estudiado con fines diversos que tratan de integrar técnicas de recogida de datos e interpretaciones tanto cuantitativas como cualitativas. Aun así, basta con partir de la premisa de que las representaciones sociales son las formas a través de las cuales construimos y deconstruimos el mundo que nos rodea para comprender que estas dan sentido a nuestras experiencias y guían nuestra toma de decisiones.

Estas representaciones, por tanto, tienen un valor teórico en diferentes campos del conocimiento (p. ej., la psicología, la sociología, la educación, entre otros) debido a que dicho concepto plantea una convergencia teórica entre aspectos cognitivos y afectivos, así como en 


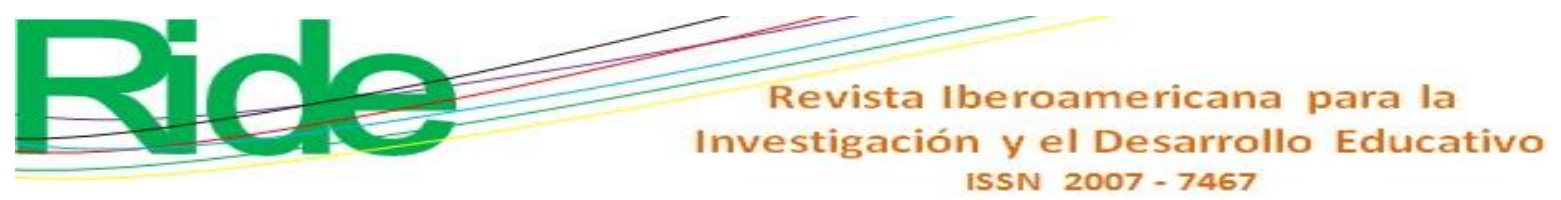

las disposiciones para actuar, lo cual permite presentar un esfuerzo sólido para integrar otros conceptos, tales como actitudes, creencias, estereotipos, etc. (Corvalán, 2013).

En ese sentido, las representaciones sociales son también una manera de entender el conocimiento que se produce, pues supera la dicotomía individuo-sociedad, dado que la propuesta parte de prácticas compartidas que hacen sentir a los sujetos que sus conductas no son azarosas, sino que parten de conocimiento verdadero. Al respecto, Farr (1983, citado por Corvalán, 2013) señala que las RS tienen una doble función:

Primero, establecer un orden que permita a los individuos orientarse en su mundo material y social y dominarlo; segundo, posibilitar la comunicación entre los miembros de una comunidad proporcionándoles un código para el intercambio social y un código para nombrar y clasificar sin ambigüedades los diversos aspectos de su mundo y de su historia individual y grupal (p. 119).

Partiendo de esa idea, todos conformamos y necesitamos representaciones sociales para dar sentido a la vida, pues esto nos permite racionalizar por qué nos comportamos como lo hacemos y explicar nuestra individualidad, de modo que una reflexión profunda de cómo se conforman nuestras RS (a través de qué, quiénes, cuándo y dónde) sirve para generar un diálogo y una confrontación con nuestra esencia individual. Esto se debe a que las RS también nos ayudan a entender, explicar e incluso aceptar cuáles son los roles que nos corresponden, así como la posición, a veces fatídica, que el “destino” nos coloca.

En tal sentido, prácticamente todos los conceptos (vida, amor, muerte, mérito, fracaso) han sido socialmente construidos, por lo que se puede decir que "se sostiene explícita o implícitamente una perspectiva compleja de reconocimiento de las unidades de análisis. Lo individual, lo social, lo biológico, lo cultural, lo racional, lo emocional (...) constituyen cualidades esenciales e históricas del devenir humano" (Corvalán, 2013, p. 120). Como Moscovici (1969) lo señala, "las representaciones sociales son sistemas cognitivos que tienen una lógica y un lenguaje propios, y que no son simples 'opiniones sobre', o ‘imágenes de’ o 'actitudes hacia', sino ‘teorías' sui generis destinadas a descubrir la realidad y su ordenación” (p. 35), de ahí que su estudio y análisis en profundidad sigan siendo relevantes en nuestro mundo cotidiano, pues al observar una representación social también se puede apreciar cómo se conforma, define y separa a un grupo de otros (Di Giacomo, 1987). 


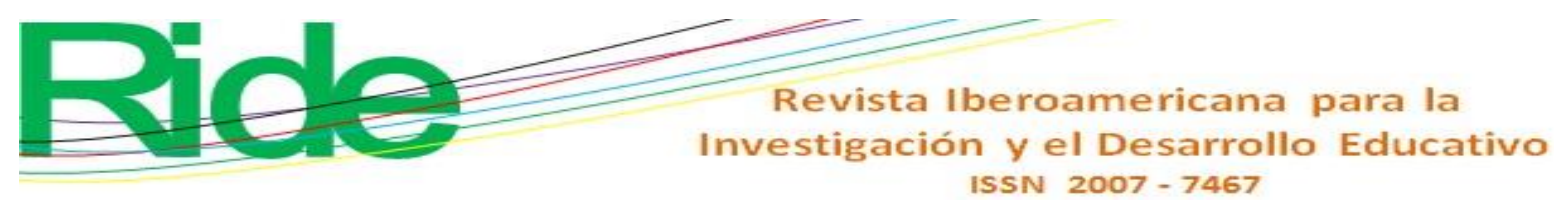

\section{Desarrollo}

En México el cuerpo de docentes es cada vez más amplio. De acuerdo con datos del Instituto Nacional de Estadística y Geografía (Inegi) y la Secretaría de Educación Pública (SEP), en 2013 existían en el país un total 236973 centros de trabajo, en los que laboraban 1266574 profesores que atendían a 23562183 estudiantes de preescolar, primaria, secundaria y centros de educación especial, lo que en términos generales indica que en el país hay al menos un profesor por cada 18 estudiantes.

Esto significa no solo que cada día se requiere un número importante de profesionales de la educación, sino también que es necesario indagar en la manera en que estos se ubican como grupo, es decir, qué hacen, dicen, comparten, estudian, etc., pues son ellos quienes interpretan y aplican el currículo vigente. Al respecto, Piña y Cuevas (2004) opinan:

Los agentes educativos se integran en comunidades en donde cultivan formas de pensamiento cercanas o apartadas de los lineamientos académicos legítimos. Un plan de estudios podrá estar finamente diseñado porque tomó en cuenta las demandas sociales del momento, las demandas de la disciplina que se enseña y la coherencia entre los contenidos y su secuencia; sin embargo, los actores educativos, maestros, estudiantes y autoridades son quienes se encargarán de instrumentarlo. Por ejemplo, las creencias de algunos de ellos sobre lo que es la formación ideal, o sobre la forma de enseñar, o bien la familiaridad con los contenidos pueden propiciar giros en el diseño de ese plan de estudios (p. 105).

En ese sentido, las representaciones sociales se crean en el propio seno de la formación del profesorado que vincula el saber generado por el sentido común (lo que $a$ priori sabe de ser maestro) con lo que técnica y científicamente se espera de él, siempre tomando en consideración que a un docente lo forma otro docente; por ello, se puede asegurar que mucho de lo que subyace en las RS son el producto de lo aprehendido de otros, quienes a su vez lo aprendieron y aprehendieron de otros. En palabras de Cruz (2006), el mundo se construye mediante un conocimiento socialmente compartido en el que influyen las interpretaciones que los demás ofrecen de la "realidad". 
Esto no quiere decir que las RS sean una especie de suerte inevitable (salvo que el sujeto lo crea de esa manera), aunque en términos generales tampoco se puede señalar que son estáticas, pues el sujeto que las produce también participa de su construcción, reconstrucción y transformación para transmitirlas a los demás "en un proceso dialéctico entre realidad objetiva y subjetiva (...) con el fin de clasificar un objeto social y explicar sus características, para incorporarlo a su realidad cotidiana” (Cruz, 2006, p. 37).

Aunado a ello, el poder de las RS se halla en que, por una parte, se incorporan al discurso individual y colectivo como referencias del deber ser y hacer, y una vez vinculadas a las prácticas cotidianas "se convierten en verdades para el sentido común” (Billig, 1993, citado por Cruz, 2006, p. 38). Esto explica por qué un grupo de profesores, por ejemplo, puede compartir percepciones similares sobre lo que sería el "buen" o el "mal" desempeño de un grupo, lo cual puede ser heredado por los siguientes profesores del ciclo escolar, quienes crearán prejuicios sobre cuál debería ser el trato que tendría que recibir determinado grupo, así como establecer qué se puede o no esperar del él. En pocas palabras, las RS si bien son colectivas, también se imbrican en una subjetividad que las transforma, utiliza y hace parte de su identidad. Por eso, Akkerman y Meijer (2011, citados por Antunes y Monereo, 2016) señalan lo siguiente en relación con la identidad profesional docente:

Es interdependiente y se configura por la percepción del maestro de sí mismo, y también por cómo es percibido por otros. Cada contexto educativo proporciona elementos para generar posturas específicas del Yo y oportunidades de aprendizaje; el aprendizaje, en este contexto, es visto como la capacidad del maestro para ajustar sus posiciones o para crear nuevas posiciones al participar en prácticas compartidas (...). Desde el punto de vista del yo dialógico, el ser maestro es un proceso de negociación continua de múltiples posiciones del Yo, de tal manera que se mantiene un sentido más o menos consistente del yo a lo largo de su vida profesional (p. 2).

Esta idea, sin embargo, cobra un matiz diferente si consideramos la identidad profesional desde lo planteado por Jodelet (1984), quien señala que el sujeto no accede a las interacciones cotidianas de forma espontánea y neutral, sino que ya cuenta con RS que le 


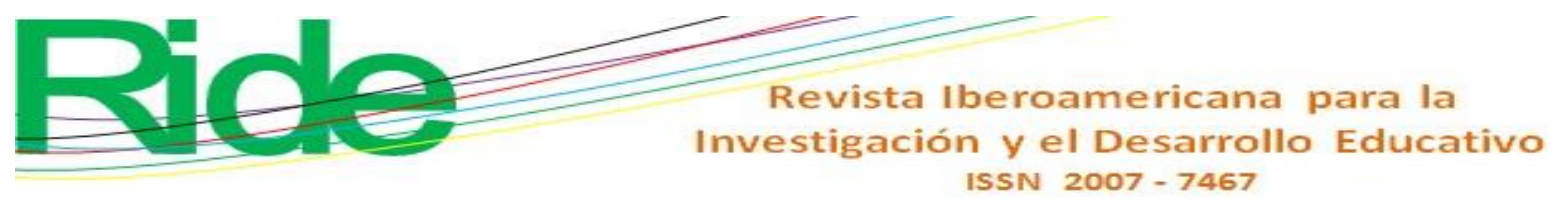

permiten anticiparse a la situación, preparar la interacción y actuar.

Por lo anterior, la teoría de las RS, así como su vinculación con la formación profesional docente y los nuevos horizontes conceptuales que gradualmente van abonando al desarrollo de las ciencias sociales hacen que destaque "la importancia que reviste la teoría y estudio de las RS en la investigación educativa (...), [pues] se trata de una problemática emergente dentro de las ciencias sociales y, especialmente, en la investigación educativa en México" (Piña y Cuevas, 2004, p. 102).

En ese sentido, las RS no son estáticas si representan "el marco de referencia que permite la clasificación y evaluación de objetos, sujetos, relaciones, acontecimientos, situaciones, etc., a partir de categorías simples y operativas" (Cruz, 2006, p. 41). Partiendo de esa premisa, se puede indicar que existen tres razones por las que el estudio de las RS de profesores en formación contribuye al campo de las ciencias de la educación:

1. Plantea nuevos cuestionamientos sobre la formación docente y el desarrollo profesional de ellos.

2. Sitúa al currículo nacional y al modelo educativo mexicano en un contexto de nuevas demandas conceptuales y sociales (Borg y Mayo, 2001).

3. Ofrece un escenario para la discusión de las actuales políticas educativas en la regulación de maestros, profesores y escuelas.

A continuación, se explican cada una de las razones mencionadas: en primer lugar, y de acuerdo con Jiménez y Perales (2007), desde hace algún tiempo está abierta la discusión sobre hacia dónde se debe dirigir el ethos magisterial: si continuar basados en la norma y en la prescripción del qué y cómo debe hacer y pensar el docente, o transitar hacia una reestructura crítica de las demandas actuales para el profesorado, lo cual alienta el debate del cómo, porqué y para qué se debe formar a los docentes.

Así pues, en esa línea de pensamiento, la investigación de las RS relacionadas con la formación docente abona a la discusión y aporta elementos teóricos para una posible reconfiguración del currículo en la formación de profesores. De hecho, al abordar el desarrollo profesional de los docentes, estos deben considerar la consolidación de su sistema 


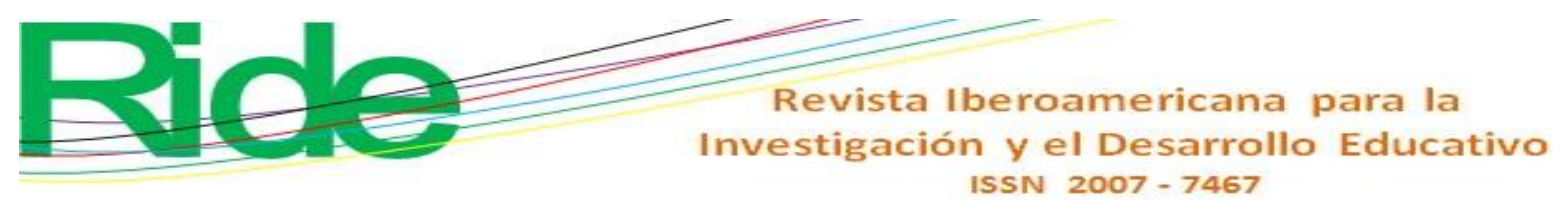

propio y, por lo tanto, de sus identidades como una actividad fundamentalmente dialógica que permita el análisis de las actividades de aprendizaje en un contexto particular.

El desarrollo profesional de los maestros suele estar representado por una lucha interna que trata de acomodar las diferentes posiciones del yo para hacerlas consistentes y continuas. Una forma de contribuir al desarrollo profesional de los profesores, desde la perspectiva dialógica, es examinar más de cerca los problemas, dilemas e incertidumbres que experimentan en las instituciones educativas diarias y en las aulas. La investigación sobre los diálogos de los profesores, y sobre sus interacciones con los estudiantes y otros actores de la escena educativa constituye un valioso medio para acceder a sus representaciones y percepciones, lo cual permitirá contribuir a su transformación y desarrollo porque brinda nuevas rutas orientadoras para formar profesores más críticos y reflexivos de su propia práctica, es decir, docentes que comprendan la magnitud de la tarea cultural que les corresponde para transformar el habitus (De Ibarrola, citado por Jiménez y Perales, 2007). Más aun, considerando que las RS de un profesor en formación lo "disponen actitudinalmente (...), marcan pautas de relaciones y de decisiones que se traducen en comportamientos, que participan en las relaciones inter e intragrupales" (Cruz, 2006, p. 38).

Adicionalmente, lo significativo de estudiar las RS radica en que también llegan a orientar las políticas públicas. Como mencionan Marambio, Gil de Montes y Valencia, (2015), si existe la creencia de que solamente algunas personas pueden alcanzar un máximo potencial de inteligencia, entonces no se necesitaría invertir recursos en personas que — dada su limitada capacidad - no serán capaces de aprovecharlas; en consecuencia, las políticas educativas serán reticentes a corregir desigualdades sociales. En cambio, si existe la creencia de que todas las personas tienen el potencial para alcanzar niveles superiores de inteligencia, esto orientará iniciativas educativas más favorables y equitativas (Rattan et al., 2012, citados por Marambio et al., 2015). En otras palabras, las representaciones de inteligencia están asociadas al éxito o al fracaso escolar, pues generalmente se juzga en mayor o menor medida que el estudiante inteligente es exitoso y, por tanto, meritorio. Sobre este aspecto, Marambio et al. (2015) comentan: 
La justificación de que solo los más inteligentes puedan acceder a recursos educativos, a su vez, argumenta jerarquías sociales y fomenta valores basados en el mérito personal y en la competitividad. Por lo tanto, el pensamiento científico construido en torno a la inteligencia es una fuente de autoridad, legitimación y de justificación de las decisiones político-deológicas (Wagner \& Halles, 2011). Creencias como la meritocracia, tan conocida en el ámbito académico, está compuesta por una concepción de inteligencia individual. Si la persona no posee capacidades genéticamente dadas y se esfuerza por superar sus limitaciones, la responsabilidad del desarrollo de la inteligencia recae en el individuo. De esta manera, si hay éxito académico, el estudiante es merecedor de acceder a mejores oportunidades en la vida, lo que será un premio a su esfuerzo personal. En un sentido contrario, el fracaso escolar es atribuido a la falta de dedicación del estudiante, por lo que no tendrá acceso a oportunidades de desarrollo escolar y, por ende, a mejores oportunidades laborales a futuro (p. 7).

Por lo anterior, estudiar las RS de éxito y fracaso también repercute en las políticas educativas y, en el caso particular de la presente investigación, en materia de formación de profesores, pues el currículo está determinado por la Secretaría de Educación. Por ello, el objetivo de este trabajo fue analizar las representaciones sociales de éxito y fracaso en estudiantes universitarios que cursan la carrera de docencia.

\section{Metodología}

En Latinoamérica y Europa existe una vasta literatura en materia de representaciones sociales, la cual sirve como sustento para asegurar que dicho constructo está conformado por elementos constitutivos y constituyentes. Parafraseando a Corvalán (2013), en el concepto representaciones sociales se halla implícita la necesidad de definir lo psicosocial, tanto de lo individual como de la forma en la que el colectivo funciona, es decir, según los elementos biológicos, sociales, individuales y culturales que lo constituyen. Esta heterogeneidad ocasiona que desde el punto de vista metodológico se deba prever que también existen “distintos métodos de abordaje para el estudio de las representaciones sociales, distintas 
técnicas cuantitativas, técnicas cualitativas, que son desplegadas en función de las distintas vertientes teóricas (...) [que dependen] del enfoque teórico-metodológico que propongan" (Corvalán, 2013, p.118).

Tomando en cuenta esta particularidad, la presente indagación pretende seguir aportando fundamentos teóricos y críticos que sirvan para nutrir el estudio de las RS desde otras miradas metodológicas. En concreto, y para recabar los datos, se diseñó un instrumento para el análisis de representaciones sociales de profesores en formación, el cual estuvo constituido por una escala de diferencial semántico que se les presentó a los participantes con el fin de determinar los constructos planteados: alumno exitoso o fracasado.

Los adjetivos que constituyeron la escala fueron extraídos de la revisión de la literatura, así como del modelo propuesto por Kaplan (1992), que divide en diferentes categorías los vocablos sugeridos con el propósito de evidenciar si hacia alguno de estos se inclina la concepción del profesorado en formación, o lo que Perrenoud denominó oficio de ser alumno. Cabe señalar que los ítems 3, 6, 11,13, 14, 17, 18 y 19 fueron recodificados para que su procesamiento fuera consistente con los demás pares de palabras del instrumento.

\section{Resultados}

En este trabajo se contó con la colaboración de un grupo de 42 estudiantes para profesor de una universidad del sureste de México. De estos, 19 (45.2 \%) fueron hombres y $23(54,8 \%)$ fueron mujeres. La edad media de los participantes fue de 20 años. El promedio escolar del grupo, considerando las calificaciones del semestre anterior, fue de 87.56 puntos. Vale señalar que $33.3 \%$ de los participantes indicó haber reprobado entre 1 y 6 asignaturas a lo largo de su trayecto formativo de licenciatura.

Estas variables fueron importantes debido a que en futuros momentos se pretenden realizar diferentes análisis comparativos a partir de dichas variables. En una primera contextualización de los participantes, y a partir de la interrogante ¿Cuál es el máximo nivel educativo al que aspiras?, es posible apreciar lo siguiente (tabla 1): 
Tabla 1. Sexo y nivel educativo

\begin{tabular}{lllllll}
\hline \multicolumn{7}{c}{ ¿Cuál es el máximo nivel educativo al que aspiras? } \\
& Licenciatura & Especialidad & Maestría & Doctorado & Total \\
\hline Sexo & Hombre & 3 & 3 & 4 & 9 & 19 \\
& Mujer & 0 & 4 & 10 & 9 & 23 \\
\hline Total & 3 & 7 & 14 & 18 & 42 \\
\hline
\end{tabular}

Fuente: Elaboración propia

Como se puede apreciar en la tabla 1, no se presentan variaciones importantes en relación con el sexo y las aspiraciones de los participantes en cuanto a una mayor preparación académica. No obstante, sí existe una correlación entre la reprobación de asignaturas y el número de estas en relación con las aspiraciones académicas, como se nota en la tabla 2:

Tabla 2. Relación entre aspiraciones académicas y asignaturas reprobadas

\begin{tabular}{|c|c|c|c|}
\hline & & & $\begin{array}{r}\text { ¿Cuál es el } \\
\text { máximo nivel } \\
\text { académico al que }\end{array}$ \\
\hline & & ¿Has reprobado? & aspiras? \\
\hline \multirow[t]{2}{*}{ ¿Has reprobado? } & Correlación de Pearson & 1 & $.307 *$ \\
\hline & Sig. (2-tailed) & & .048 \\
\hline \multirow{2}{*}{$\begin{array}{l}\text { ¿Cuál es el máximo nivel } \\
\text { académico al que aspiras? }\end{array}$} & Correlación de Pearson & $.307 *$ & 1 \\
\hline & Sig. (2-tailed) & .048 & \\
\hline \multirow[t]{2}{*}{ ¿Cuántas has reprobado? } & Correlación de Pearson & & $-.350 *$ \\
\hline & Sig. (2-tailed) & & .023 \\
\hline
\end{tabular}

Fuente: Elaboración propia 

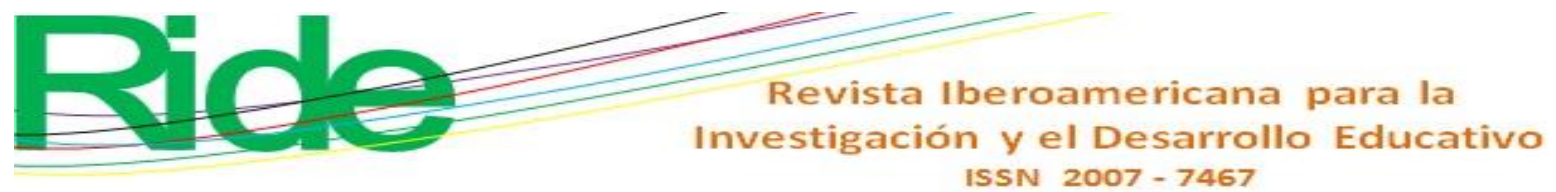

Como se puede observar, el valor $p$ está por debajo del alfa esperado (0.005), por lo que en ambos casos resulta relevante. Sin embargo, vale indicar que aunque la correlación no es tan fuerte, es un primer indicador de cómo los éxitos y fracasos pueden, eventualmente, determinar cuánto se pretende seguir avanzando. Evidentemente, no es el único factor, pero sí es una variable que no se puede desestimar. De hecho, se puede notar la correlación negativa entre el nivel de aspiraciones y el número de asignaturas reprobadas, pues en la medida en que estas aumentan, disminuye el nivel educativo que se pretende alcanzar. Nuevamente, esto coincide con la idea de que éxitos y fracasos construidos en la escuela y desde la escuela pueden ser determinantes en ciertos ideales, pues perpetúan la idea de que quien avanza tiene méritos, potencial y capacidades que lo hacen más apto para la vida académica.

Por otra parte, en cuanto a los elementos que deben caracterizar a un estudiante exitoso, los participantes respondieron lo siguiente (tabla 3): 


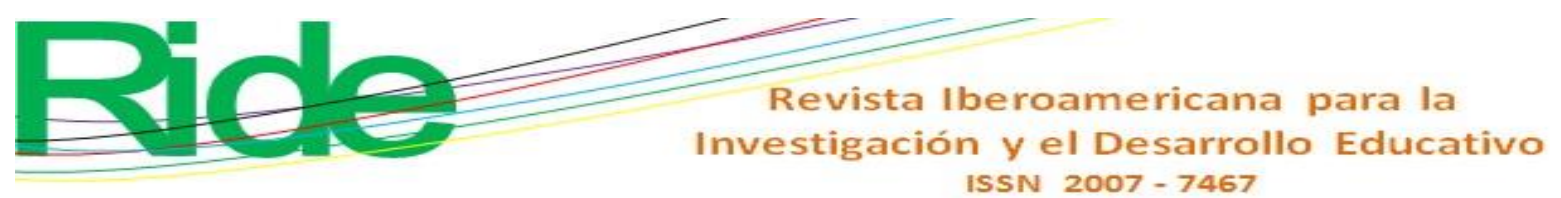

Tabla 3. Respuestas de la RS: alumno exitoso

\begin{tabular}{lccccc}
\hline & & & & & $\begin{array}{c}\text { Desviación } \\
\text { estándar }\end{array}$ \\
\hline Familia separada-familia & 42 & 4 & 7 & 4.07 & .463 \\
constituida & & & Máximo & Media & \\
Reservado-conversador & 42 & 2 & 7 & 4.24 & .983 \\
Inquieto-tranquilo & 42 & 1 & 7 & 4.33 & 1.373 \\
Agresivo-dócil & 42 & 3 & 7 & 4.55 & .889 \\
Sociable-aislado & 42 & 2 & 7 & 5.19 & 1.401 \\
Desaseado-aseado & 42 & 2 & 7 & 5.21 & 1.440 \\
Simpático-antipático & 42 & 3 & 7 & 5.21 & 1.220 \\
Lento-ágil & 42 & 2 & 7 & 5.33 & 1.262 \\
Grosero-amable & 42 & 1 & 7 & 5.55 & 1.485 \\
\hline Inteligente & 42 & 4 & 7 & 5.69 & 1.070 \\
\hline Maduro-inmaduro & 42 & 2 & 7 & 5.69 & 1.259 \\
\hline Puntual-impuntual & 42 & 1 & 7 & 5.74 & 1.363 \\
\hline Tradicional-innovador & 42 & 2 & 7 & 5.79 & 1.220 \\
\hline Apático-interesado & 42 & 2 & 7 & 5.79 & 1.240 \\
\hline Cuidadoso-descuidado & 42 & 4 & 7 & 5.79 & 1.048 \\
\hline Atento-distraído & 42 & 4 & 7 & 5.95 & 1.058 \\
\hline Torpe-hábil & 42 & 4 & 7 & 6.20 & 1.030 \\
\hline Acrítico-crítico & 42 & 7 & 6.29 & 1.043 \\
\hline Holgazán-trabajador & 4 & 7 & 6.38 & .962 \\
\hline Motivado-desmotivado & 4 & 7 & 6.57 & .914 \\
\hline Irresponsable-responsable & 42 & 7 & 6.40 & .857 \\
\hline & 42 & & & & \\
\hline
\end{tabular}

Fuente: Elaboración propia

Una de las ventajas que ofrece el diferencial semántico se halla en que (en contraste con otro tipo de escalas como la de tipo Likert) propone un continuo de significados cuyas propiedades psicométricas permiten identificar distancias entre los opuestos de palabras, y no solo un orden determinado, es decir, es posible dar un tratamiento de escala de intervalo y obtener medidas de tendencia central y de desviación. 

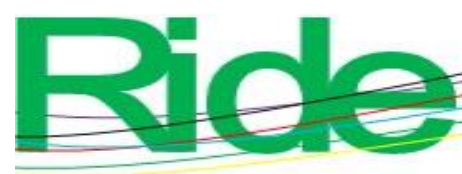

Revista Iberoamericana para la Investigación y el Desarrollo Educativo ISSN $2007-7467$

Por lo anterior, y siendo el diferencial de siete puntos en la escala, el valor 4 representaría la neutralidad entre los pares polares de adjetivos. En ese sentido, tomemos los extremos que constituyen la RS del alumno exitoso. En primer término, podemos apreciar que las características de familia separada o constituida (entendiendo a esta última como familia tradicional, bajo los esquemas hegemónicos heternormativos) fue el elemento menos valorado como un factor que pudiera ser determinante en el éxito del estudiante. De hecho, presentó la menor desviación estándar, es decir, fue mucho más consistente la respuesta generalizada de los sujetos de estudio.

Este dato coincide con la literatura y el propio modelo de Kaplan (1992), quien encontró que para los maestros mexicanos, a diferencia de otros profesores de Latinoamérica, no era tan importante la constitución familiar, como tampoco lo era que el estudiante fuera reservado o conversador.

Por el contrario, y de acuerdo con la percepción de los participantes, la RS de un alumno exitoso está constituida por las siguientes cualidades: ser responsable $(x=6.57, \mathrm{DS}$ $=.914)$, estar motivado $(\mathrm{x}=6.40, \mathrm{DS}=.857)$ y ser trabajador $(\mathrm{x}=6.38, \mathrm{DS}=.962)$; es decir, la idea de éxito se asocia con características intrínsecas en el alumno, esto es, propias del esfuerzo individual y la persistencia, así como de la capacidad para alentarse de manera autónoma.

En relación con el fracaso, los resultados parecen indicar que se seleccionaron los mismos tres pares de palabras para categorizar al alumno que fracasa, aunque en diferente orden y puntuación: desmotivado $(\mathrm{x}=2.40, \mathrm{DS}=1.499)$, irresponsable $(\mathrm{x}=2.43, \mathrm{DS}=$ 1.343) y holgazán ( $\mathrm{x}=2.60, \mathrm{DS}=1.106)$; cabe señalar que los datos a la inversa indican que en la medida que el puntaje se acerca a 1, la oposición del atributo es "favorable".

Igualmente, es interesante subrayar que es mayor la dispersión de los datos en relación con el fracaso, lo cual se puede explicar debido a los múltiples elementos que se pueden considerar en torno al bajo rendimiento del alumno. Aun así, los resultados generales de la caracterización de fracaso se presentan en la tabla 4: 


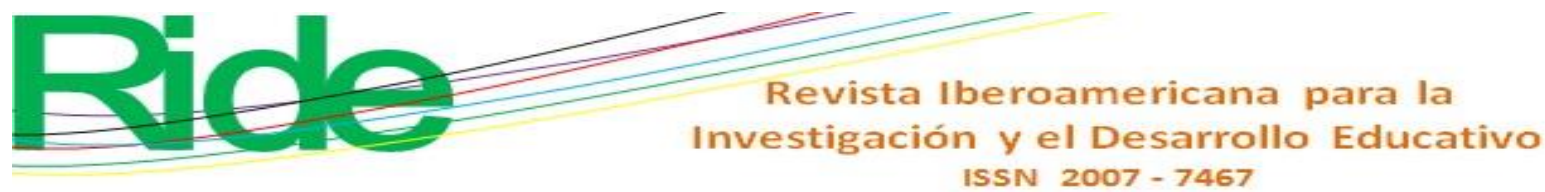

Tabla 4. Respuestas de la RS: alumno que fracasa

\begin{tabular}{|c|c|c|c|c|c|}
\hline & $\mathrm{N}$ & Mínimo & Máximo & Media & Desviación \\
\hline Motivado-desmotivado & 42 & 1 & 7 & 2.40 & 1.499 \\
\hline Irresponsable-responsable & 42 & 1 & 7 & 2.43 & 1.346 \\
\hline Holgazán-trabajador & 42 & 1 & 4 & 2.60 & 1.106 \\
\hline Atento-distraído & 42 & 1 & 5 & 2.69 & 1.259 \\
\hline Puntual-impuntual & 42 & 1 & 4 & 2.79 & 1.260 \\
\hline Cuidadoso-descuidado & 42 & 1 & 6 & 2.88 & 1.214 \\
\hline Acrítico-crítico & 42 & 1 & 7 & 2.90 & 1.265 \\
\hline Maduro-inmaduro & 42 & 1 & 6 & 3.07 & 1.156 \\
\hline Apático-interesado & 42 & 1 & 7 & 3.07 & 1.455 \\
\hline Lento-ágil & 42 & 1 & 6 & 3.48 & 1.087 \\
\hline Tradicional-innovador & 42 & 1 & 6 & 3.57 & 1.151 \\
\hline Simpático-antipático & 42 & 1 & 7 & 3.67 & 1.408 \\
\hline Inquieto-tranquilo & 42 & 1 & 6 & 3.67 & .874 \\
\hline Torpe-hábil & 42 & 1 & 7 & 3.67 & 1.282 \\
\hline Grosero-amable & 42 & 1 & 7 & 3.69 & 1.024 \\
\hline Inteligente & 42 & 2 & 4 & 3.71 & .596 \\
\hline Agresivo-dócil & 42 & 1 & 6 & 3.74 & .939 \\
\hline Reservado-conversador & 42 & 1 & 6 & 3.79 & 1.025 \\
\hline $\begin{array}{l}\text { Familia separada-familia } \\
\text { constituida }\end{array}$ & 42 & 1 & 4 & 3.88 & .504 \\
\hline Sociable-aislado & 42 & 1 & 7 & 4.07 & 1.197 \\
\hline Desaseado-aseado & 42 & 1 & 7 & 4.12 & 1.109 \\
\hline
\end{tabular}

Elaboración propia 


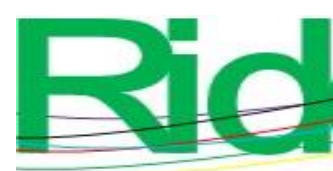

\section{Conclusión}

Los resultados hallados en esta investigación permiten concluir que existen ciertos consensos en la representación social que un grupo de estudiantes universitarios que cursan la carrera de docencia tiene en torno a las características que definen a un alumno exitoso o que fracasa. En la figura 1 se pueden observar las semejanzas y diferencias encontradas:

Figura 1. Comparación de medias entre los pares de alumno exitoso y fracasado

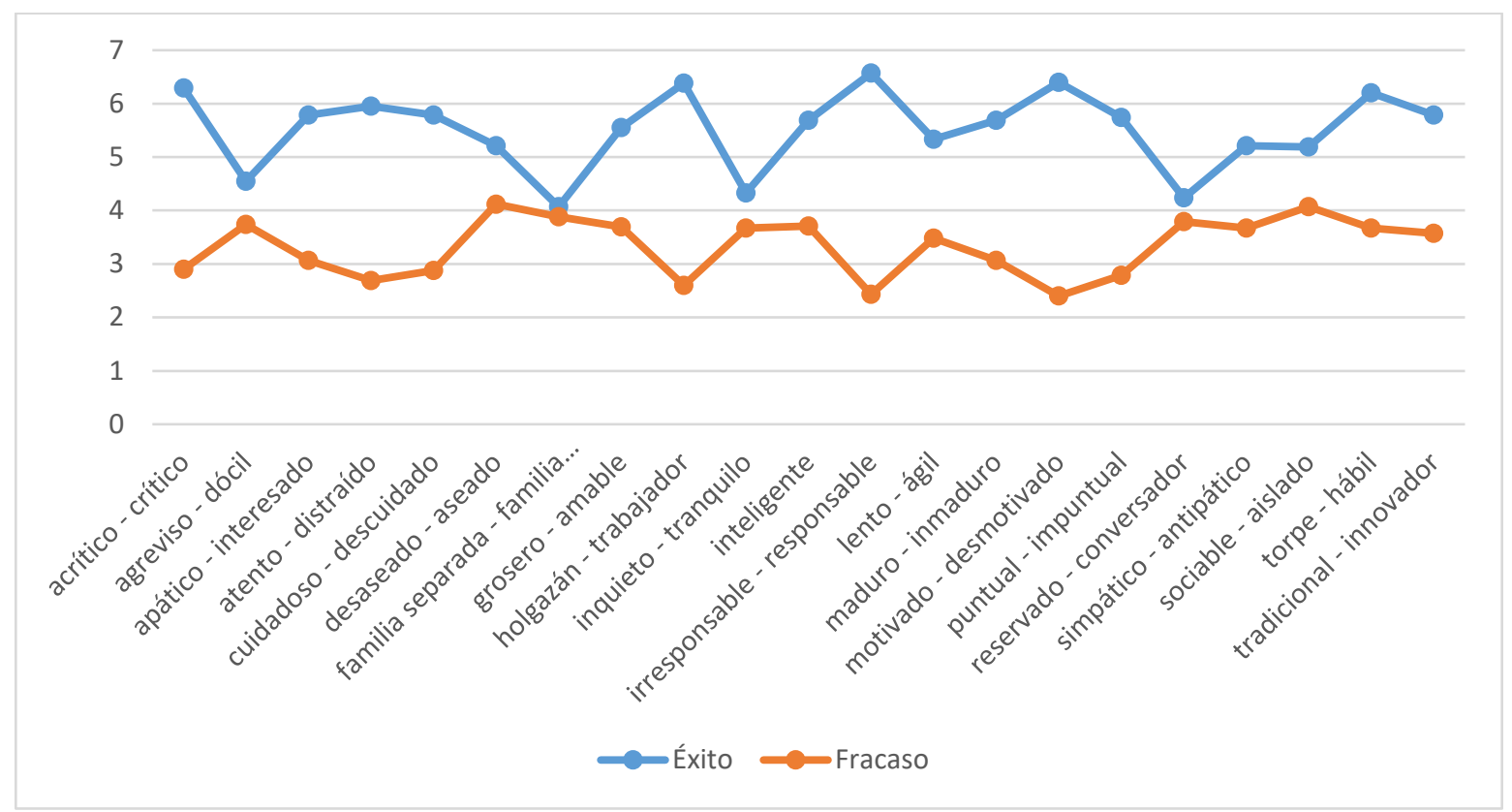

Fuente: Elaboración propia

En la figura 1 se aprecia mayor diferencia entre las palabras responsable, trabajador y motivado, y más cercanía (mínima diferencia) entre la constitución familiar y el comportamiento en el aula del alumno (en términos de si es callado o conversador). Asimismo, llama la atención el calificativo inteligente, pues este — desde la visión del alumno - es un elemento neutral para que una persona fracase, aunque sí lo es para conseguir el éxito ( $\mathrm{x}=5.69)$, aunque se halla por debajo del trabajo y el esfuerzo. 

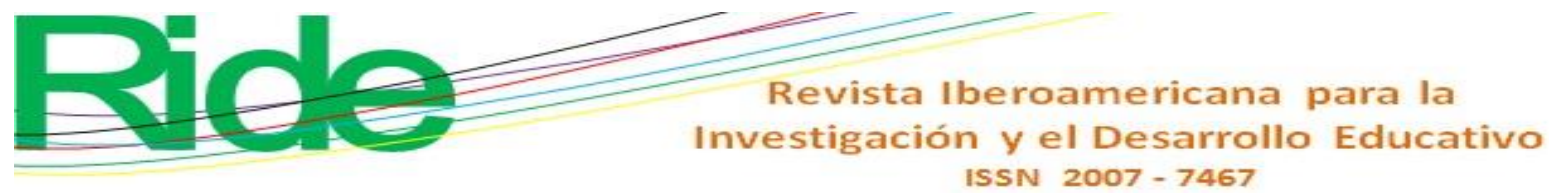

Según lo anterior, se puede afirmar que los estudiantes tienen una visión del éxito y del fracaso orientada al esfuerzo continuo y al trabajo perseverante, por lo que se esperaría que actuaran en consecuencia. Estos resultados ofrecen nuevas posibilidades para seguir indagando en este fenómeno, el cual se puede comparar con otras poblaciones y maneras de entender dichos conceptos. De esta manera, se puede contribuir a un entendimiento más profundo tanto del quehacer del profesorado como de otras variables, como el sexo, el plan de estudio, el semestre, entre otras. Además, estos hallazgos en conjunto podrían servir para la generación de políticas educativas para docentes y estudiantes. En el caso de los primeros, en materia de formación del profesorado, promoción y educación continua; en el caso de los estudiantes, para acceso a becas y otros niveles educativos. Todo esto, de hecho, puede servir para brindar más elementos en torno a la manera en que son evaluados y reciben retroalimentación, lo cual puede transparentar aún más el proceso para visibilizar posibles vicios y retos, así como para fomentar una auténtica justicia social a través del proceso educativo. 


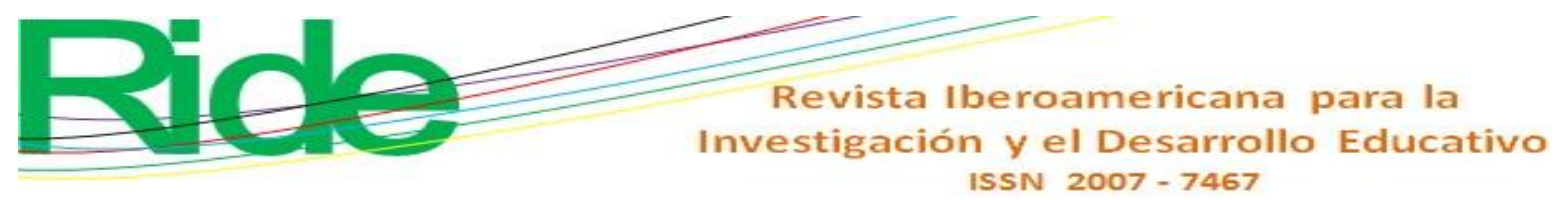

\section{Referencias}

Antunes, R. y Monereo, C. (2016). The Development of University Teachers' Professional Identity: A Dialogical Study. Research Papers in Education, 33(1), 42-58.

Borg, C. and Mayo, P. (2001). Social difference, cultural arbitrary and identity: An analysis of a new national curriculum document in a non-secular environment. International Studies in Sociology of Education, 11(1), 63-86. Doi: 10.1080/09620210100200067.

Corvalán, F. (2013). 50 años de representaciones sociales y psicología: campo psy, bifurcaciones y desafíos. Estudos Contemporâneos da Subjetividade, 3(1), 115-127. Recuperado de http://www.periodicoshumanas.uff.br/ecos/article/viewFile/1055/823.

Cruz, F. (2006). Género, psicología y desarrollo rural: la construcción de nuevas identidades. España: Serie Estudios.

Di Giacomo, J. P. (1987). Teoría y método de análisis de las representaciones sociales. En Páez, D. (ed.), Pensamiento, individuo y sociedad. Cognición y representaciones (pp. 278-295). Madrid: Fundamentos.

Jiménez, M. y Perales, F. (2007). Aprendices de maestros. La construcción de sí. México: Pomares.

Jodelet, D. (1984). La representación social: fenómenos, conceptos y teoría. En Moscovici, S. (ed.), Psicología social II. Pensamiento y vida social. Psicología social y problemas sociales (pp. 478-494). Barcelona: Paidós.

Kaplan, C. (1992) Buenos y Malos alumnos. Descripciones que predicen (4 $\left.{ }^{\mathrm{a}} \mathrm{ed}\right)$. Argentina: AIQUE.

Marambio, K., Gil de Montes, L. y Valencia, J. (2015). Representaciones sociales, inteligencia y conflicto de la educación en Chile. Psykhe, 24(1), 1-11. Doi: https://dx.doi.org/10.7764/psykhe.24.1.643.

Moscovici, S. (1969). The phenomenon of social representations. In Farr, R. M. and Moscovici, S. (eds.), Social representations (pp. 3-69). Cambridge, University Press.

Piña, J. y Cuevas, Y. (2004). La teoría de las representaciones sociales. Su uso en la investigación educativa en México. Perfiles Educativos, 26(105), 102-124.

Secretaría de Educación Pública (SEP) (2013). Principales cifras del sistema educativo $\begin{array}{llll}\text { mexicano. } & \text { México. }\end{array}$ 
Revista Iberoamericana para la Investigación y el Desarrollo Educativo ISSN $2007-7467$

http://planeacion.sep.gob.mx/estadisticaeindicadores.aspx.

\begin{tabular}{|c|c|}
\hline Rol de Contribución & Autor (es) \\
\hline Conceptualización & Galo López Gamboa \\
\hline Metodología & Galo López Gamboa \\
\hline Software & Galo López Gamboa \\
\hline Validación & Edith Cisneros Cohernour \\
\hline Análisis Formal & Galo López Gamboa \\
\hline Investigación & Galo López Gamboa (principal) Martín Aguilar Riveroll (apoyo) \\
\hline Recursos & Edith Cisneros Cohernour (igual) Martín Aguilar Riveroll (igual) \\
\hline Curación de datos & Galo López Gamboa (principal) Edith Cisneros Cohernour (apoyo) \\
\hline $\begin{array}{l}\text { Escritura - Preparación del borrador } \\
\text { original }\end{array}$ & Galo López Gamboa \\
\hline Escritura - Revisión y edición & $\begin{array}{l}\text { Galo López Gamboa (principal)Edith Cisneros Cohernour (apoyo) } \\
\text { Martín Aguilar Riveroll (apoyo) }\end{array}$ \\
\hline Visualización & Galo López Gamboa (igual) Edith Cisneros Cohernour (igual) \\
\hline Supervisión & Galo López Gamboa \\
\hline Administración de Proyectos & Edith Cisneros Cohernour \\
\hline Adquisición de fondos & Martín Aguilar Riveroll (igual) Edith Cisneros Cohernour (igual) \\
\hline
\end{tabular}

